\title{
IMMUNOHISTOCHEMICAL LOCALIZATION OF ELASTIC SYSTEM FIBRES IN THE CANINE PROSTATE
}

\author{
Marettová, E. \\ Department of Anatomy, Histology and Physiology \\ University of Veterinary Medicineand Pharmacy, Komenského 73, 04181 Košice \\ Slovakia \\ elena.marettova@uvlf.sk
}

\section{ABSTRACT}

The elastic fibres are particularly important for the structural integrity and function of the prostate. In this study, the elastic fibres of the normal dog prostate gland were identified by immunohistochemistry. In the capsule, the elastic fibres form membranes of different thicknesseslocated mainly in the intermediate and deep zones. Large trabeculae which extend from the capsule contain elastic fibres with a prevalence in the longitudinal direction. Around blood vessels, the elastic fibres are concentrated and form annular structures. In the fine septa supporting the lobules,elastic fibres form a fine elastic meshwork. Between the secretory units of the prostate gland,the fine elastic fibres are located under the secretory epithelium. An increase of elastic fibres around the ducts entering the urethra was observed. An accumulation of elastic fibres in the capsule and stromal septa may participate in the releasing of secretory products during ejaculation.

Keywords: dog; elastic fibres; immunohistochemistry; prostate

\section{INTRODUCTION}

The presence of elastic tissue as a normal component of the stromal tissue has been reported ingenito-urinary organs. Most studies have been related to the urinary organs. In the female canine urethra,the elastic fibres system was described by Augsburger [1] and in the vesicourethral junction and urethra of the guinea pig by Dass et al. [2]. Murakumo et al. [11] in their scanning electron microscopic study,observed three dimensional arrangement of collagen and elastin fibres in the human urinary bladder. Elastin was also found in association with the human male membranous urethral sphincter [7].

Prostatic disorders are accompanied by extensive but poorly understood modifications of the cells and surrounding extracellular matrix. In the human prostate, the distribution of the elastic system fibres in prostatic disorders have been reported [15] and a positive correlation between the tumour progression and the presence of elastic fibres in the tumour stroma was found [8]. The elastin as a stromal component was increased in benign prostatic hyperplasia patients [12]. The elastic components in these cases were of 
variable thicknesses and formed a three-dimensional network at the base of the epithelium. These results indicate, that prostate cancer cell invasion involves extensive remodelling of the fibres of the elastic system.

Studies on the occurrence of elastic fibres in the accessory glands in animals are scarce. By immunohistochemical methods elastic fibres were localized in the bovine vesicular gland [9].The presence of elastic fibres in the prostate is generally accepted, but their distribution is not well known. Dimitrov [4] described the elastic fibres in the prostate gland of the cat and Delella and Felisbino [3] in the rat. Little is known about the presence and arrangement of the elastic fibrillary components of the stroma of the normal prostate in the dog. Therefore, the aim of this work was to study the distribution of elastic fibres in the supporting tissue of the dog prostate by immunohistochemical means.

\section{MATERIALS AND METHODS}

Tissue samples of the prostate glands from five clinically healthy adult dogs (German Shepherd, age 3 to 4 years) have been used in this study. Samples of the tissue were fixed in $10 \%$ neutral formalin in $0.2 \mathrm{~mol}$ phosphate buffer for $24 \mathrm{~h}$, then washed, dehydrated, cleared in xylene, and thereafter routinely embedded in paraffin for histological and immunohistochemical studies.

\section{Immunohistochemistry}

Sections $5 \mathrm{~mm}$ thick were collected on silanized glass slides, dewaxed with xylene, and rehydrated in a descending ethanol series. Immunohistochemistry was performed by the streptavidin-biotin peroxidase method. Endogenous peroxidase activity was blocked by treating the tissue sections with $3 \%$ hydrogen peroxide in methanol for $30 \mathrm{~min}$. Nonspecific protein-protein interactions were blocked by incubating the sections with 3\% Bovine Serum Albumin (BSA) (Sigma) in phosphate-balanced salt solution (PBS) containing $0.1 \%$ Tween 20 for 1 hour. Monoclonal antibodies against elastin (Sigma, 1:5000) were used. The primary antibodies were diluted in $1 \%$ BSA in PBS, and incubated overnight at $4^{\circ} \mathrm{C}$. The sections were then washed and incubated with a secondary peroxidase-conjugated antibody. After washing with PBS, the peroxidase activity was visualized with $0.05 \% 3^{\prime} .3^{\prime}$-diaminobenzidine and $0.03 \%$ v/v $\mathrm{H}_{2} \mathrm{O}_{2}$. The slides were counterstained with Mayer's haematoxylin, air dried,and mounted in Entellan (Merck, Darmstadt, Germany). Negative controls were performed by omitting the primary antibodies.

\section{RESULTS}

Histologically, the prostate gland is a tubuloalveolar gland and consists of two portions: the external (compact) and internal (disseminate). The capsule and stromal septa of the prostate consists of fibrous connective tissue in which smooth muscle cells, fibroblasts, collagen and elastic fibres have been identified.

Immunohistochemically, in the capsule elastic tissue reactive to elastin antibody occupy the intermediate and inner zones and consists of elastic membranes and less of fibres (Fig. 1).In the large septa arising from the capsule and those between the lobules the elastic fibres form a dense elastic meshwork (Fig. 2) with a prevalence of the fibres in the longitudinal direction. Elastic fibres in different amounts are in the interlobular septa and in increased number around the blood vessels (Fig. 3). Between the secretory units of the prostate gland the elastic fibres were seen to form an elastic meshwork, whereas between the secretory alveoli elastic fibres they are arranged longitudinally (Fig. 4). In the wall of stromal arteries, the elastic fibres were barely present. A concentration of elastic fibres was found around the ducts and in the area of the urethra (Fig. 5).

\section{DISCUSSION}

The stroma of the prostate gland is dynamic tissue which directly influences the behaviour of the epithelium and is activated in response to injuries $[6,13,14]$. Previous studies have suggested that elastic tissue is a normal component in the genito-urinary region $[1,11]$. Our results showed that the capsule of the dog prostate contain a large number of elastic membranes of different thicknesses forming one thick layer and partially integrated in the muscle layer of the capsule. In the stroma of the canine prostate, like in the cats [4], the elastic fibres were observed with almost equal density in the different parts of the glandular interstices. The elastic fibres and membranes were observed mainly in large interlobular septa, where they were intermingled with 


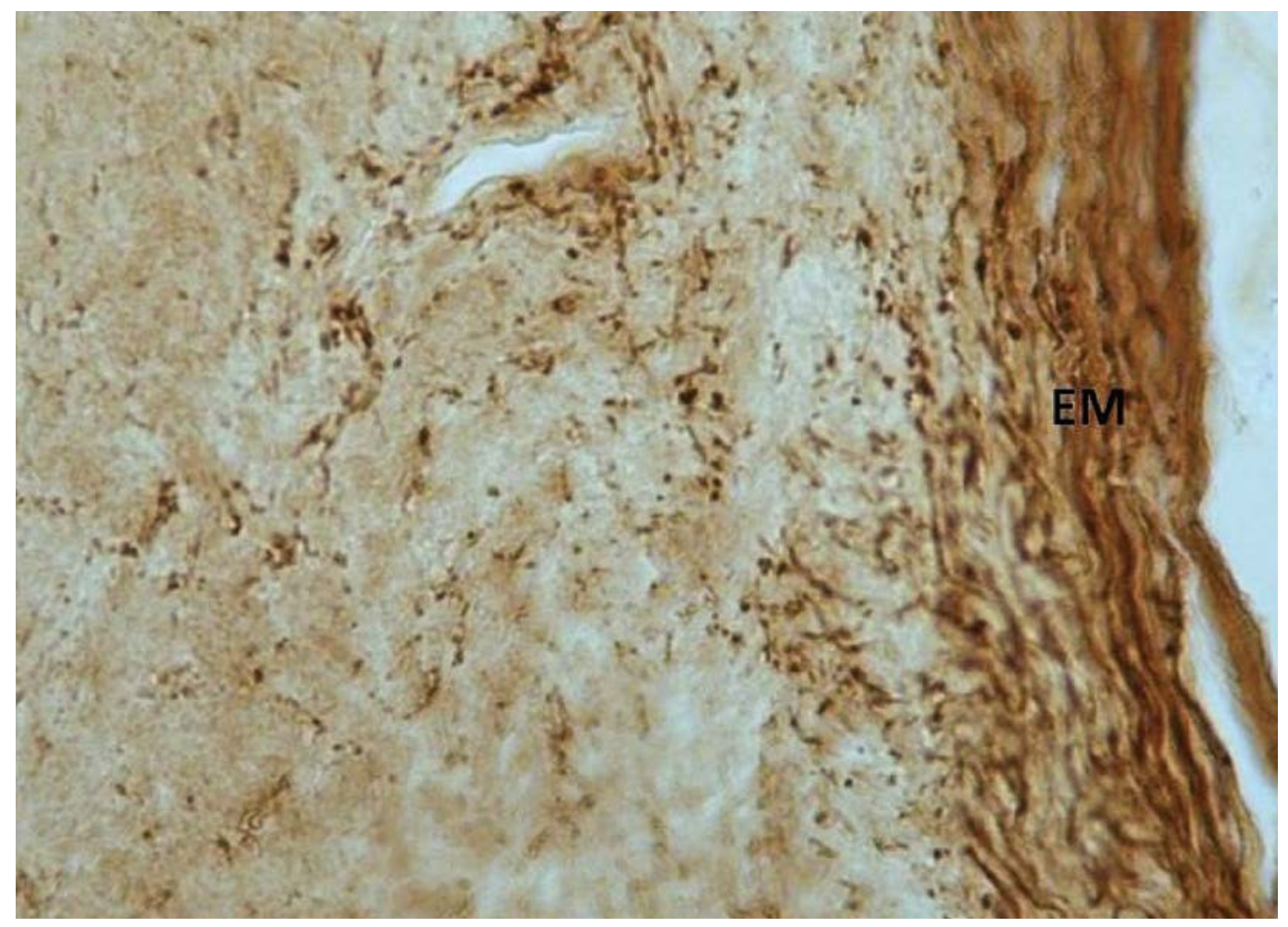

Fig. 1. Capsule and underlying tissue of the prostate. The capsule revealed elastic thick membranes (EM). Magn. $\times 450$

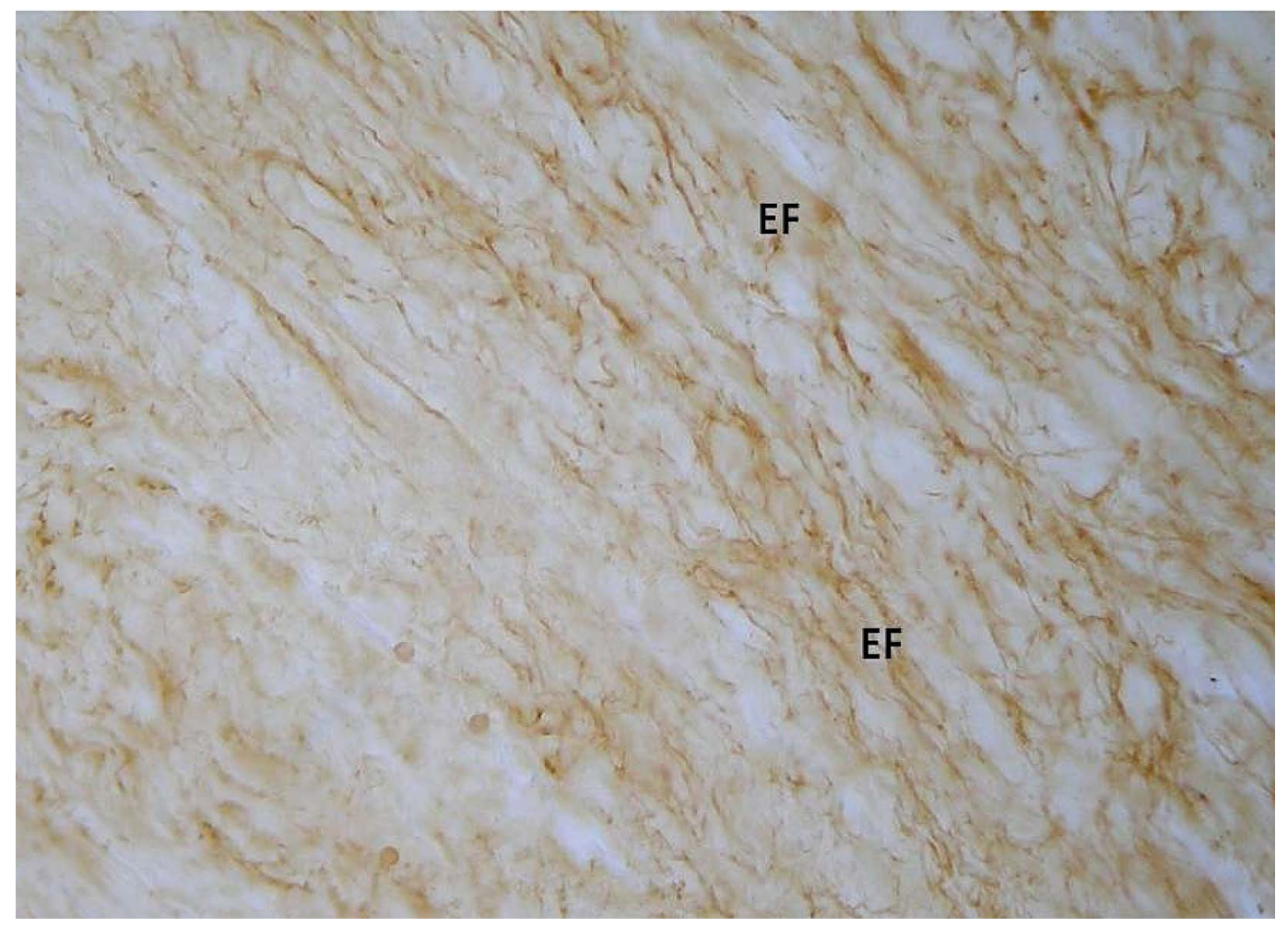

Fig. 2. Section of the large trabeculae. Elastic fibres (EF) form a dense meshwork with a prevalence in the longitudinal direction. Magn. $\times 450$ 


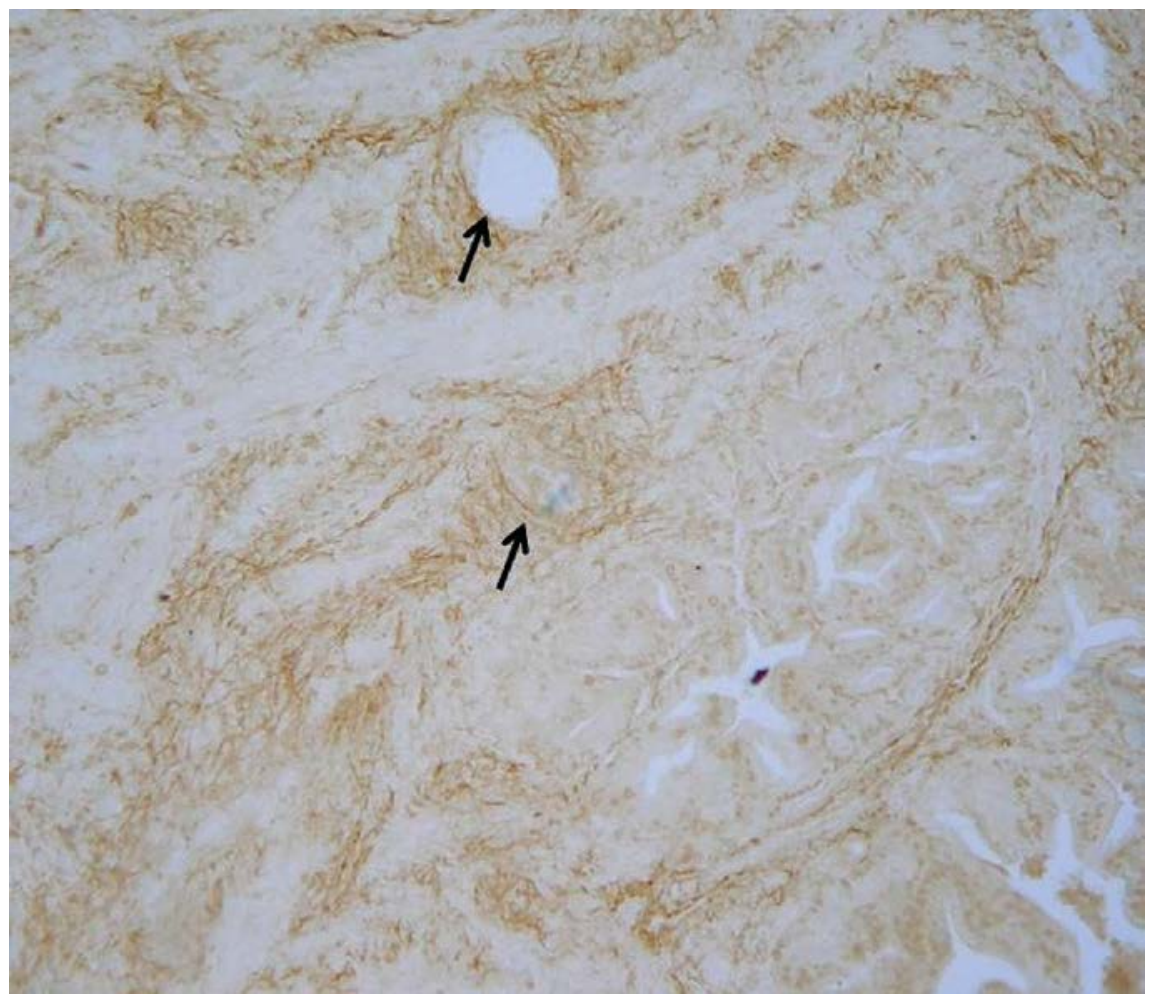

Fig. 3. Section of prostatic lobule. Elastic fibres in different amount are increased around blood vessels (arrows). Magn. $\times 200$

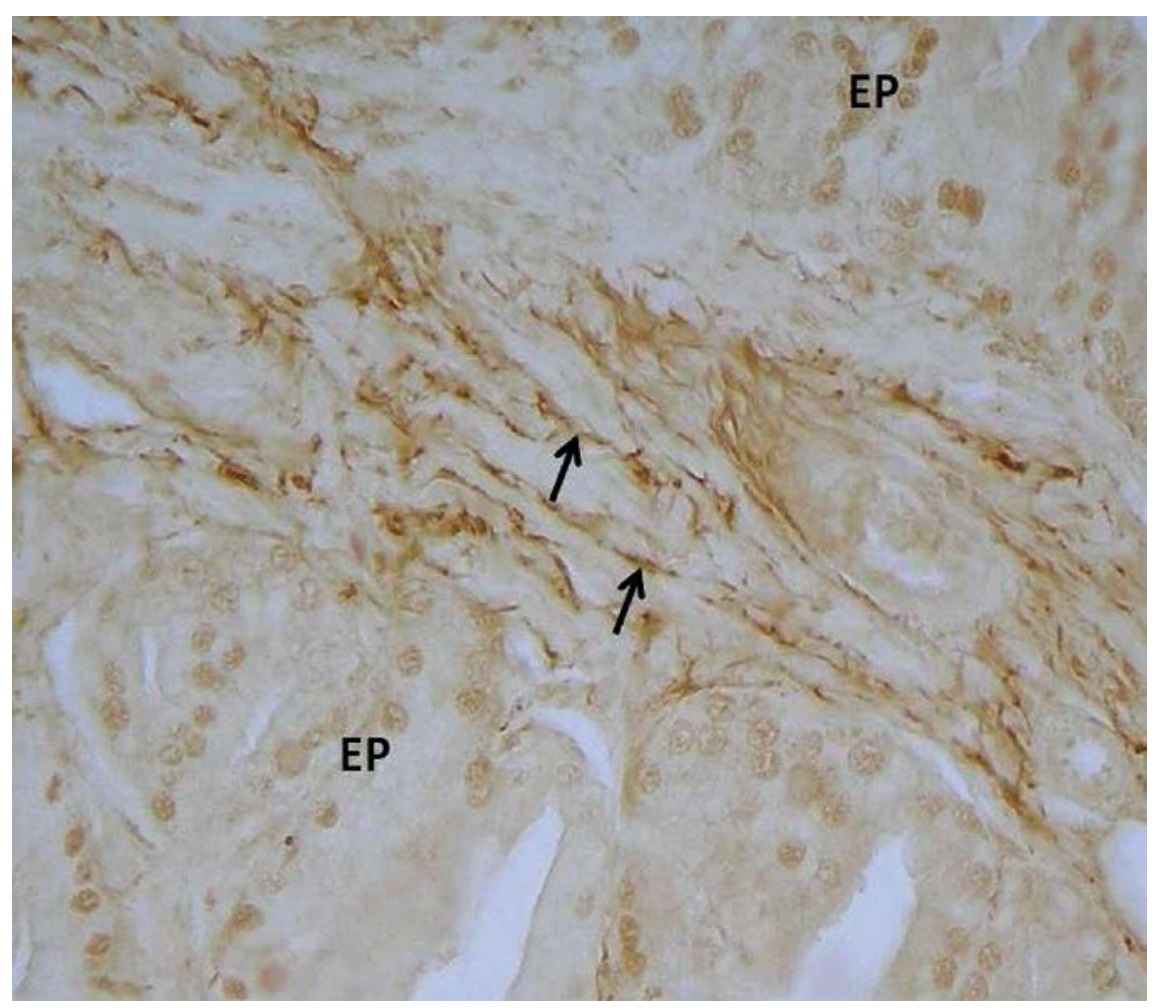

Fig. 4. Section of prostatic lobule. An accumulation of elastic fibres and membranes (arrows) among the secretory alveoli with secretory epithelium (EP). Magn. $\times 1000$ 


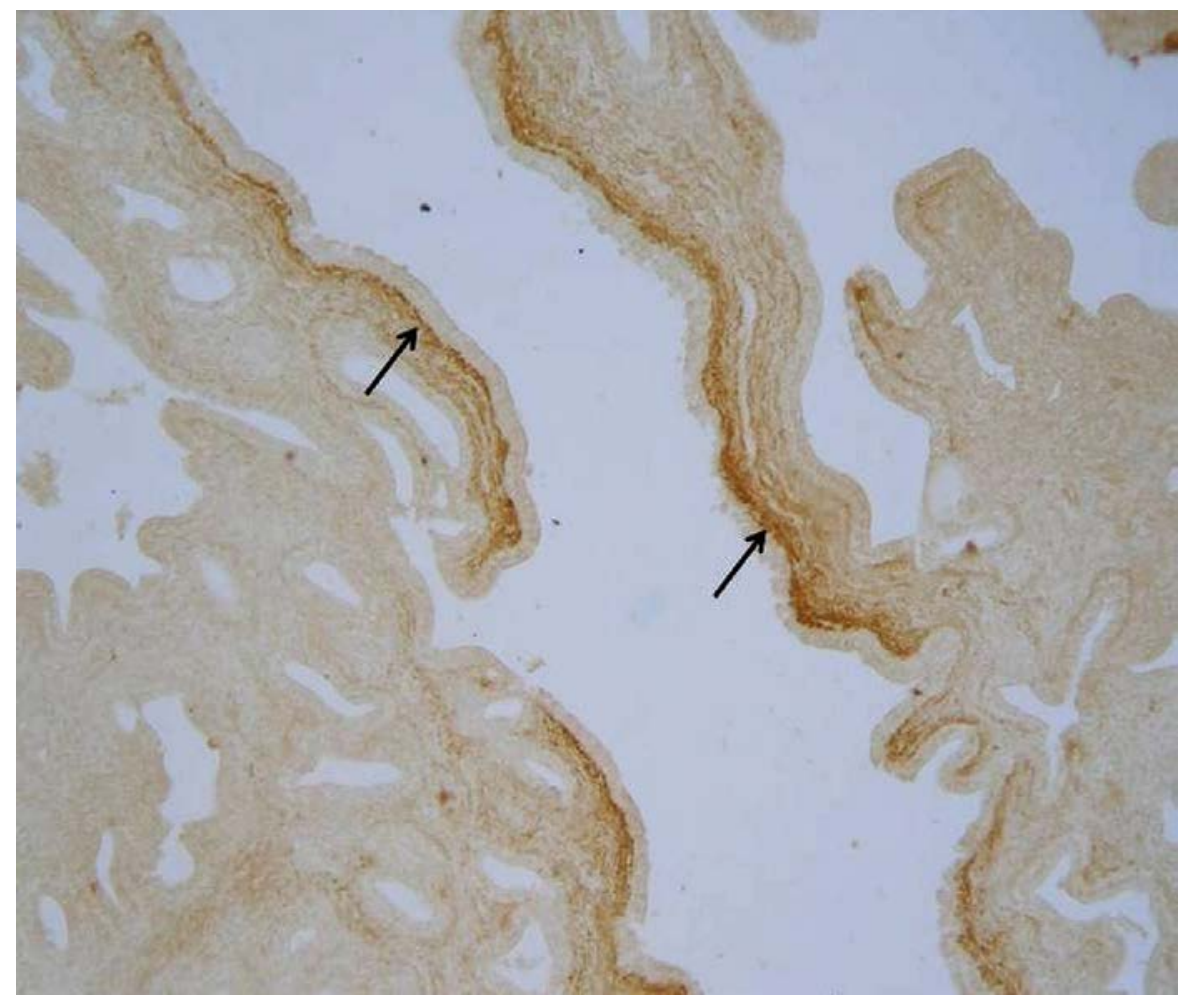

Fig. 5. Prostatic duct opening into prostatic portion of the urethra.

An accumulation of elastic fibres below the lining epithelium (arrows). Magn. $\times 200$

smooth muscle cells. Among the secretory alveoli, elastic fibres are involved in a concentric fibrous extracellular matrix surrounding the normal acini. The stroma undergirds the acinar epithelium through its fibromuscular substance [5]. In the rat prostate, the elastic fibres in this area were thin and inconspicuous as was described [3].

An accumulation of elastic fibres was observed in the connective tissue in the area of the urethra. A similar increase of elastic fibres was reported in the vesicourethral junction and urethra of the guinea pig [2] and also in the cat [4]. An abundance of elastic fibres amongst the smooth muscle bundles associated with the human male membranous urethral sphincter has been reported by $\mathrm{Ho}$ et al. [7]. The fibres were seen to be associated with the longitudinal smooth muscle bundles of the urethra and urinary bladder of the guinea pig [10,11]. Das s et al. [2] considered the elastic fibres associated with the smooth muscle bundles of the urethra suitable for both uniting the contractile force of the smooth muscle bundles to shorten the urethra, as well as augmenting passive recoil of the longitudinal smooth muscle bundles, hence restoring the urethral in length.
The number and organization of elastic tissue change in the case of benign prostatic hyperplasia where the larger proportion of elastin in the area of the urethra and at the base of prostate was noted [12]. In benign prostatic hyperplasia the elastic components were of variable thickness and formed a three-dimensional network at the base of the epithelium [15]. An increased variability in the elastic fibre distribution was observed in adenocarcinomas, depending on the tumour grade. In adenocarcinomas with little differentiation, in some hyperplasic acini, and in the stroma adjacent to tumour masses, ruptured and residual elastic fibres indicative of matrix degradation or remodelling were seen. In more undifferentiated tumours, a preelastic network, perhaps indicative of a new extracellular matrix microenvironment was seen [15]. These results indicate that prostate cancer cell invasion involves extensive remodelling of the fibres of the elastic system. According to Delella and Felisbino [3], the deposition of collagen and elastic system fibres in the prostatic stroma may counterbalance the absence of smooth muscle tone during a-blockers treatment. 
Although it is supposed that elastic fibres in the prostatic connective tissue are predominantly mechanical, they may also have a specific function. The elastic fibres may play a role in the resistance of these components in the prostate during secretory activity and in restoration after expulsion of prostate secretion following ejaculation. An accumulation of elastic fibres present around the external layer of blood vessels may be involved in this action. According to W e in berg et al. [16], the ability of elastin-rich structures to deform and subsequently recover depends on the interactions of elastin with other fibrillary components and with proteoglycans and non-collagenous glycoproteins.

\section{CONCLUSIONS}

The results of this study revealed that the capsule and stroma of the dog prostate was rich in elastic tissue. The capsule of the dog prostate contained a large number of elastic membranes of different thicknesses forming one thick layer and partially integrated in the muscle layer of the capsule. In the stroma of the canine prostate, the elastic fibres were observed with almost equal density in the different parts of the glandular interstices. Among the secretory acini, elastic fibres were involved in concentric fibrous extracellular matrix.

\section{REFERENCES}

1. Augsburger, H.R., 1997: Elastic fibres system of the female canine urethra. Histochemical identification of elastic, elaunin and oxytalan fibres. Anat. Histol.Embryol., 26, 297-302.

2. Dass, N., McMurray, G., Brading, A.F., 1999: Elastic fibres in the vesicourethral junction and urethra of the guinea pig. J. Anat., 195, 447-453.

3. Delella, F. K., Felisbino, S. L., 2010: Doxazosin treatment alters stromal cell behaviour and increases elastic system fibres deposition in rat prostate. Micr. Res. Tech., 73, 1036-1044.

4. Dimitrov, R., 2010: Localization and distribution of elastic fibres in the stroma of accessory sex glands and the wall of pelvic urethra in male cats. Trakia J. Sci., 8, 83-88.
5. Farnsworth, W.E., 1999: Prostate stroma: physiology. Prostate, $38,60-72$.

6. Grossfeld, G. D., Hayward, S. W., Tlsty, T. D., Cunha, G. R., 1998: The role of stroma in prostatic carcinogenesis. Endocr. Relat. Cancer, 5, 253-270.

7. Ho, K. M. T., Dass, N., Brading, A. F., Noble, J., 1997: Elastin in association with the human male membranous urethral sphincter. Neurourol. Urodyn., 16, 454-455.

8. Lapis, K., Tímár, J., 2002: Role of elastin-matrix interactions in tumour progression. Semin. Cancer Biol., 12, 209-217.

9. Marettová, E., Legáth, J., 2010: The presence of smooth muscle cells and elastic fibres in the bull vesicular gland. Slov. Vet. Res., 47, 91-96.

10. Murakumo, M., Ushiki, T., Koyanagi, T., Abe, K., 1993: Scanning electron microscopic studies of smooth muscle cells and their collagen fibrillary sheaths in empty, distended and contracted urinary bladders of the guinea pig. Arch. Histol. Cytol., 56, 441-449.

11. Murakumo, M., Ushiki, T., Abe, K., Matsumura, K., Shinno, Y., Koyanagi, T., 1995: Three dimensional arrangement of collagen and elastin fibres in the human urinary bladder: a scanning electron microscopic study. J. Urol., 154, 251-256.

12. Sugimoto, K., Matsumoto, S., Uemura, H., Ito, H., 2008: Distribution of elastic fibers on prostate. Hinyokika kiyo. Acta Urol. Japonica, 54, 321-324.

13. Tuxhorn, J. A., Ayala, G. E., Rowley, D. R., 2001: Reactive stroma in prostate cancer progression. J. Urol., 166, 2472-2483.

14. Tuxhorn, J. A., Ayala, G. E., Smith, M. J., Smith, V.C., Dang, T. D., Rowley, D. R., 2002: Reactive stroma in human prostate cancer: induction of myofibroblast phenotype and extracellular matrix remodelling. Clin. Cancer Res., 9, 2912-2923.

15. Vilamaior, P.S.L., Suzigan, S., Carvalho, H.F., Taboga, S.R., 2003: Structural characterization and distribution of elastic system fibers in the human prostate and some prostatic lesions. Braz. J. Morphol. Sci., 20, 101-107.

16. Weinberg, P.D., Winlove, C.P., Parker, K.H., 1995: The distribution of water in arterial elastin: effects of mechanical stress, osmotic pressure, and temperature. Biopolymers, 35, $161-169$.

Received November 3, 2016

Accepted December 5, 2016 